\title{
Staging Neurological Disorders: Expressions of Cognitive and Motor Disorder
}

\author{
Trevor Archer • Richard M. Kostrzewa
}

Received: 31 October 2009/Revised: 2 November 2009/Accepted: 2 November 2009/Published online: 26 November 2009

(C) Springer Science+Business Media, LLC 2009

\begin{abstract}
In neurologic disorders, there are progressive losses in regional brain structural integrity, circuitry, and neuronal process that threaten individuals' ability to express functional capacity at several levels of severity. The classification of (a) patients on the basis of diagnosis, risk prognosis, and intervention outcome forms the basis of clinical staging and (b) laboratory animals on the basis of animal model of brain disorder, extent of insult and dysfunctional expression, provides the components for the clinical staging and preclinical staging, respectively, of the disease state with certain associated epidemiological, biological, and genetic characteristics. The investigation of epigenetics and biomarkers is intrinsic to any analysis of the progressive nature of the neurogenerative disorders, in the present account disorders relating to Alzheimer's disease, Parkinson's disease, depression, and diabetes.
\end{abstract}

Keywords Staging - Braak - Alzheimer disease . Parkinson disease $\cdot$ Depression $\cdot$ Diabetes

The "Staging" of neurological disorders has been adapted and, to some extent, modified from the applications that are intrinsic to disease diagnosis, prognosis, and treatment in oncology. Patients are classified as low-, medium-, or highrisk based on a combination of clinical staging of the disease and certain epidemiological, biological, and genetic

T. Archer $(\square)$

Department of Psychology, University of Gothenburg,

Box 500, 40530 Gothenburg, Sweden

e-mail: trevor.archer@psy.gu.se

R. M. Kostrzewa

Department of Pharmacology, Quillen College of Medicine,

East Tennessee State University, Johnson City, TN 37614, USA characteristics such as the age of the patient, extent of disease spread, microscopic appearance, and genetic factors. Brain structure and function in health/illhealth presents a dynamic, energized equation incorporating neurochemistry, neuronal processes, and circuitry components. In neurologic disorders, there are progressive losses in all of these components, and as a consequence, the integrity of brain structure is compromised, with a deterioration that is ordered, reproducible, and stepwise. For example, the Hoehn and Yahr (1967) Staging of Parkinson's Disease includes: Stage I presenting mild, unilateral symptoms and signs, inconvenient rather than disabling, with usually tremor of one limb, and noticeable changes in posture, walking and facial expression; Stage II presenting bilateral symptoms with minimal disability affecting posture and gait; Stage III presenting a marked slowing of body movements with impairment of equilibrium on walking or standing and moderately severe generalized dysfunction; at Stage IV, there are presented severe symptoms with only limited walking, rigidity and bradykinesia, and inability to survive alone; and finally, Stage V presents a "Cachetic" stage of complete invalidism with inability to stand/walk, requiring constant nursing care. Hoehn and Yahr Staging system has been supplanted by the more complex Unified Parkinson's Disease Rating Scale that incorporates sections pertaining to (a) 'Mentation, Behavior and Mood, (b) Activities of Daily living and Speech, and (c) Motor examinations with speech. The order offered through the clinical staging of neurological disorders was examined and explained in the presentations at the most recent Fundacion Cerebro y Mente Meeting in Mojacar, Spain, "Staging Neuropsychiatric Disorders: Implications for Etiopathogenesis and Treatment", October 14-18, 2009.

K.A. Jellinger (presentation delivered by P. Riederer) outlined a comprehensive longitudinal evaluation of the 
cognitive decline that is the major symptom of Alzheimer's disease (AD), by application of standardized instruments to illustrate the steady, yet varying, advance in disease progression, from the pre-dementia and very mild dementia levels, exemplified by mild cognitive impairment (MCI) to the advanced, terminal level. The Braak staging in AD as well as in other neurodegenerative conditions which involves neuroimaging, metabolic, and neurochemical biomarkers provide stages in the Alzheimer disease continuum. "Progressions" through the Braak Stages benchmark "Regressions" in cognitive functioning. Notwithstanding the exceptions to certain Braak stages, e.g., timing of synaptic loss, the progression of the neurodegenerative changes, A-beta $(\mathrm{A} \beta)$ deposits, etc., appears well correlated with the clinical status. Staging provides for the early detection and continued progression of $\mathrm{AD}$ thereby facilitating development and application of therapeutic strategies despite the lack of sufficient understanding of the plethora of multiple structural brain changes. $\mathrm{K}$. Ebmeier described studies involving pre-dementia, mild dementia, and dementia using comparisons between MCI and healthy controls, and measurements of episodic memory, e.g., HVLT total recall and delay, semantic memory, and attentional/executive functioning processes through identification of patients with MCI, depressive symptoms, and AD. They found that the outcome of threefourths of the MCI patients may be predicted by performances on a comprehensive cognitive screening measure (ACE total) and a measure of delayed word recognition (HVLT-R). The following differences in performance levels were obtained: Healthy controls $>$ Depressives $>$ MCIs $>$ ADs. Amyloid ligand binding using $\left[{ }^{11} \mathrm{C}\right] \mathrm{PIB}$-PET analyses-indicated extensive amyloid labeling in the brains of AD patients. Several aspects of staging criteria in $\mathrm{AD}$ were enumerated, including: institutionalization or nursing home, clinical psychiatric setting (neuropsychology-psychiatry-neurology), amyloid status, hippocampal size, functional status, etc. The role of these various imaging markers in the staging of $\mathrm{AD}$ was discussed. J.L. Whitwell observed that the progressive development of $\mathrm{AD}$ over presymptomatic-prodromaldementia phases, temporally and chronologically, from a viewpoint of clinical and pathological progression, provided a means of understanding staging of the disorder. She described the application of longitudinal MRI as a powerful tool allowing the assessment of brain deterioration progression over multiple imaging time-points with numerous applications in neurodegenerative dementias. Several voxel-level techniques have described time-based regional atrophy in temporal, parietal, and frontal regions, particularly during the prodromal phase. It appears that atrophy of neural tissue begins in the medial temporal lobes and fusiform gyrus at least 3 years prior to the $\mathrm{AD}$ diagnosis, then spreading to the posterior temporal and parietal lobes; at the time of the first diagnosis of $\mathrm{AD}$ the pathology develops a greater severity and widespread pattern encompassing the medial temporal lobe, the temporoparietal cortices, and the frontal lobe. These patterns of atrophy were shown to correlate with the clinicopathological progression of neurofibrillary tangles with an accelerating rate. Braak et al. (2006) have described six stages in AD, the so-called Braak Stages 1-6. Full clinical dementia appears at about Braak 4. If we take the mid-50s as an index age, the odds of being at Braak 1 or beyond are about $35 \%$, Braak 2 or beyond-12\%, Braak 3 or beyond $-4 \%$. By 70 years of age the figures become: Braak 1 or beyond $-70 \%$, Braak 2 or beyond-45\%, Braak 3 or beyond-26\%. It is now estimated that the process that finally appears as dementia may well begin before age 40 (MacDonald 2007). According to the Braak hypothesis, alpha-synucleinopathy (AS) initially affects the medulla oblongata and progresses to more rostral brain areas in a hierarchical sequence with strong involvement of the amygdala, common in older individuals with clinical phenotypes ranging from normal neuropsychological status through movement disorders to dementia. ASs occur pathophysiologically from the disruption of select groups of nuclei to pervasive degeneration throughout the neuraxis. P.G. Ince et al. found that AS was common in older individuals and often associated with AD-type pathology. Half the brains studied corresponded to Braak stages 5 and 6 , and $29 \%$ corresponded to amygdala-predominant AS, but yet a large proportion of cases did not fit a hierarchical staging system. It was concluded that there was evidence that AS was more prevalent in cases with marked AD pathology, but nevertheless that did not necessarily contribute to the clinical phenotype (cf. Zaccai et al. 2006, 2008; Savva et al. 2009).

In discussing the links between cocaine dependence and substantial cognitive impairments, C.W. Bradberry addressed the relevance to staging notions from controlled animal studies performed upon macaque monkeys selfadministering, or not, cocaine. The animals were tested in a drug-free state, $72 \mathrm{~h}$ following the final administration of cocaine. A range of functional deficits was observed, these included: expressions of cognitive and motor impulsiveness and impairments in attention, planning, memory (including visual working memory), and executive control, all of which implicated regions of the cingulated cortex, orbitofrontal, and temporal cortex, as well as tasks specifically associated with the dorsolateral prefrontal cortex. There were selective impairments in cognitive flexibility, stimulus discrimination, and matching-to-sample tasks, and a significant increase in errors of omission. Chronic users of cocaine demonstrate structural deficits in cortical regions mediating many of the tasks described. The role of 
attentional processes in the performance of the tasks applied in these studies appears to be central. K. Sugaya described studies demonstrating the effects of human neural stem cell (HNSC) transplantation into the brains of aged rats which differentiated into selective neural cells and improved significantly the cognitive performances of the transplanted animals. These studies indicated that HNSCs may offer promising candidates for cell-replacement therapies in the treatment of neurodegenerative diseases. He reported also new technologies to differential adult human mesenchymal stem cells, subsets of stromal cells in bone marrow into neural cells by over-expression of the embryonic stem cell gene, nanog. The peripheral administration of a pyrimidine derivative enhanced stem cell proliferation in aged animals. These and other findings served to underline an ever-advancing strategic initiative for stem cell therapies in the neurodegenerative disorders.

Much experimental evidence indicates that several agents (e.g., nicotine) affecting the electrochemical process cause long-lasting changes (e.g., linked to long-lasting memories) in the brain associated with different components of behavior (e.g., Pascual et al. 2009). For example, it has been shown that the phosphorylation of CREB and expression of fos protein, as indicators of neural activity, accompany the acquisition and maintenance of nicotineinduced CPP but not CPA in mesolimbic areas (Nucleus Accumbens, Ventral Tegmental Area, Prefrontal cortex, and Dorsal Striatum) as well as in memory consolidation structures (hippocampus and amygdala) and nicotinic receptors are involved in this process (Pascual et al. 2009). Taken together, these studies identify the brain regions where pCREB activity is essential for nicotine preference. J.H. Medina and I. Izquierdo reported the remarkable facility of the brain in its capacity to encode and store information that induces appropriate changes in behavioral expression. In this regard, memory was presented as a temporally graded process through which transcription and translation steps must be induced during the first hours following acquistion training. Thus, they have postulated that the paradigmatic feature of long-term memory (LTM) is its persistence and yet little is known about the mechanisms that make some LTMs last longer than others. Using Inhibitory Avoidance (IA) acquisition in rats, they showed that the long-lasting fear LTM vanished rapidly when the $\mathrm{D}_{1}$ dopamine (DA) receptor antagonist SCH 23390 was injected into the dorsal hippocampus at $12 \mathrm{~h}$, but not immediately or $9 \mathrm{~h}$, after the fearful experience. On the other hand, the intrahippocampal application of the DA $\mathrm{D}_{1}$ agonist SKF 38393 at the same critical post-training time converted a rapidly decaying fear LTM into a persistent one (Rossato et al. 2009). This effect was mediated by brain-derived neurotrophic factor (BDNF) and regulated by the ventral tegmental area. It appears that a late phase of consolidation in the dorsal hippocampus is required for maintaining long-lasting (7-14 days) but not short-lived (1-2 days) long-term memory for IA in rats. They indicated that the delayed post-training phase is specifically involved in persistence but not LTM formation and is protein synthesis-, BDNF-, ERK1/2- and c-fos dependent with overall dopaminergic control. M. Cammarota maintained the notion of DA influences in persistent memories by indicating that long-lasting IA was abolished by administration of the DA $\mathrm{D}_{1}$ antagonist SCH 23390 into the dorsal hippocampus $12 \mathrm{~h}$, but not $9 \mathrm{~h}$, after IA acquisition. Interestingly, the intrahippocampus administration of the DA $\mathrm{D}_{1}$ agonist SKF 38393, at the simultaneous posttraining interval reinforced a 'swiftly' decaying LTM into a long-lasting, persistent memory; this effect was shown to be mediated by BDNF. Taken together, these presentations demonstrated that LTM persistence, or "persistent memories," were dependent upon the activation of VTA/hippocampus dopaminergic connections and modulated by manipulations performed at specific post-training intervals.

As described previously (Kostrzewa et al. 2008), the process of receptor supersensitivity (RSS) has a long history and is an epiphenomenon of denervation with DARSS being a process that is ideally suitable for examining pathophysiology in both neuropsychiatric and neurodegenerative disorders. Thus, the treatment of stereotypic progression, i.e., motor dyskinetic progression, in association with DARSS, by R.M. Kostrzewa et al. was a compelling account of a preclinical, laboratory-packaged form of disorder staging. In intact rats, the repeated administration of the $\mathrm{DA} \mathrm{D}_{2}$ agonist quinpirole evoked permanent DARSS, a "priming" phenomenon. In the neonatal destruction of DA innervations, the repeated administration of L-Dopa or DA $D_{1}$ agonists produced a highly prominent priming of $\mathrm{DA} \mathrm{D}_{1}$ receptors expressed by a progression in stereotyped behaviors and patterns of locomotor behavior that are linked to DA $\mathrm{D}_{1}$ RSS. It was indicated that alterations in DA receptor sensitization play an important role influencing interpretations of events and in the reactions to various stimuli, as expressed in the behavioral deficits shown by cocaine- and amphetamine-abusers, as well as in the disorganized thought disorders central to schizophrenia and psychosis. It was illustrated also how serotoninergic RSS modulated DARSS, thereby demonstrating the interactive nature of the neurotransmitter systems. T. Archer and A. Fredriksson maintained the theme of dopaminergic contributions to brain disorders, from a staging perspective, by showing the hypokinesic effects of 1-methyl-4-phenyl1,2,3,6-tetrahydropyridine (MPTP) in the induction of "mild," "severe," and "progressive" forms of Parkinsonism. Here, it was described how daily 30-min periods of physical exercise attenuated or reversed the bradykinesia induced by different regimes of MPTP in mice; the agent 
Milmed both reinforced and potentiated the effects of physical exercise upon motor behavior and DA-restoration. Finally, E. Rodriguez-Farre and C. Sunol discussed the neurotoxic effects, particularly upon cerebellar granular cells, of methylmercury, an important environmental contaminant. Prolonged exposure to a subcytotoxic concentration of methylmercury increased significantly nonphosphorylated cofilin, a member of the ADF/cofilin family. Cofilin is a ubiquitous actin-binding factor required for the reorganization of actin filaments. The decrease in Pcofilin induced by methylmercury was concentrationdependent and occurred after different exposure times. Since the balance between the phosphorylated and nonphosphorylated form of cofilin regulates actin dynamics and facilitates actin filament turnover, filamentous actin dynamics and reorganization are necessary for modulation of neuron shape changes, cell migration, polarity formation, regulation of synaptic structures and function, and nerve cell apoptosis.

J.M. Delgado-Garcia and A. Gruart described the unique suitability of the hippocampal CA3-CA1 synapse as an excellent experimental model for studying the interactions between short- and long-term plastic changes occurring after high-frequency stimulation of Schaffer collaterals and during the acquisition and extinction of a classical eyeblink conditioning in behaving mice, by studying the optimal intensities and inter-stimulus intervals for evoking pairedpulse facilitation (PPF) or depression (PPD) at the CA3CA1 synapse. They showed too that long-term potentiation (LTP) induced by high-frequency stimulation lasted approximately 10 days while high-frequency stimulationinduced LTP evoked an initial depression of basal pairedpulse facilitation (cf. Madroñal et al. 2009); paired-pulse depression was also induced. They showed also that TrkB receptors modulate specific phases of fear learning and amygdalar synaptic plasticity through two main phosphorylation docking sites. Studies were performed also in wildtype and transgenic mice demonstrating altered learning capacities in the genetically modified mice that offer a range of experimental models for both neuropsychiatric and neurodegenerative disorders to be further elucidated. Applications of mutant-mouse variants have provided great inroads to the further staging of $\mathrm{AD}$ [e.g., familial British dementia (FBD) and familial Danish dementia (FDD) are two autosomal dominant neurodegenerative diseases caused by mutations in the BRI (2) gene]. Garringer et al. (2009) developed transgenic mice that express wild-type and mutant forms of the BRI(2) protein, with Bri (2) knock-in mutant mice, and Bri (2) gene knock-out mice showing extensive CAA, parenchymal amyloid deposition, and neuroinflammation.

Abnormalities of the hypothalamic-pituitary-adrenal (HPA) axis are implicated in major depression; hyper- cortisolaemia has been found in typical depression and hypocortisolaemia in some studies of atypical depression. Many patients expressing depressive disorders were shown to present both hypersecretion of cortisol and its metabolites. P. Riederer discussed the associations between Diabetes Type II and not only depression, but also Parkinson's disease and AD. The combination of chronic stress with hypercortisolaemia plays a role also in the etiopathogenesis of $\mathrm{AD}$ since the brain insulin resistance condition is linked to $\mathrm{A} \beta$-accumulation and the hyperphosphorylation of tau-protein. In this regard, it ought to be noted that epidemiological studies of patient journals have implicated Diabetes Type II with risk for both AD and other neurodegenerative disorders (Ristow 2004; Li and Holscher 2007). Thus, any analysis of staging with 'diabetes-type dementia' must take into account intervention strategies based upon normalization of insulin signaling in the brain, e.g., administration of the incretin hormone glucagonlike peptide-1 (GPL-1) that enhances glucose-dependent insulin secretion and lowers blood glucose in Diabetes Type II patients (Gault et al. 2003).

The notion of staging for diagnostic, predictive, and treatment efficacy has, arguably, contributed a greater impetus to the study of the neurodenerative disorders, both in the clinical and laboratory setting, than in the understanding of brain disorders usually within the precinct of neuropsychiatry. It is possible that advances in the notions provided by incursions through epigenetics, biomarkers and brain imaging techniques have been more readily available to the former, thereby 'setting the scene' for a greater exploitation of current methodological finesse.

\section{References}

Braak H, Alafuzoff I, Arzberger T, Kretzschmar H, Del Tredici K (2006) Staging of Alzheimer disease-associated neurofibrillary pathology using paraffin sections and immunocytochemistry. Acta Neuropathol 112:389-404

Garringer HJ, Murrell J, D'Adamio L, Ghetti B, Vidal R (2009) Modeling familial British and Danish dementia. Brain Struct Funct 2009 Sept 25 [Epub ahead of print]

Gault VA, Flatt PR, O'Harte FP (2003) Glucose-dependent insulinotropic polypeptide analogues and their therapeutic potential for the treatment of obesity diabetes. Biochem Biophys Res Commun 308:207-213

Hoehn MM, Yahr MD (1967) Parkinsonism: onset, progression and mortality. Neurology 17:427-442

Kostrzewa RM, Kostrzewa JP, Brown RW, Nowak P, Brus R (2008) Dopamine receptor sensitivity: development, mechanisms, presentation and clinical applicability. Neurotox Res 14:121-128

Li L, Holscher C (2007) Common pathological processes in Alzheimer's disease and type II diabetes: a review. Brain Res Rev 56:384-402

MacDonald AB (2007) Alzheimer's disease Braak Stage progressions: reexamined and redefined as Borrelia infection transmission through neural circuits. Med Hypotheses 68:1059-1064 
Madroñal N, Gruart A, Delgado-García JM (2009) Differing presynaptic contributions to LTP and associative learning in behaving mice. Front Behav Neurosci 3:7

Pascual MM, Pastor V, Bernabeu RO (2009) Nicotine-conditioned place preference induced CREB phosphorylation and Fos expression in the adult rat brain. Psychopharmacology 207:5771

Ristow M (2004) Neurodegenerative disorders associated with diabetes mellitus. J Mol Med 82:510-529

Rossato JI, Bevilaqua LR, Izquierdo I, Medina JH, Cammarota M (2009) Dopamine controls persistence of long-term memory storage. Science 325:1017-1020
Savva GM, Zaccai J, Matthews FE, Davidson JE, McKeith I, Brayne C, The Medical Research Council Cognitive Function and Ageing Study (2009) Prevalence, correlates and course of behavioural and psychological symptoms of dementia in the population. Br J Psychiatry 194:212-219

Zaccai J, Ince P, Brayne C (2006) Population-based neuropathological studies of dementia: design, methods and areas of investigation-a systematic review. BMC Neurol 9:2

Zaccai J, Brayne C, McKeith I, Matthews F, MRC Cognitive Function, Ageing Neuropathology Study (2008) Patterns and stages of alpha-synucleinopathy: relevance in a population-based cohort. Neurology 70:1042-1048 